\title{
Sitagliptin and risk of fractures in type 2 diabetes: Results from the TECOS trial
}

\author{
Robert G. Josse MBBS, FRCP, FRCPC ${ }^{1}$ | Sumit R. Majumdar MD, $M P^{2}$ | \\ Yinggan Zheng MA, $\mathrm{MEd}^{3} \mid$ Amanda Adler MD, $\mathrm{PhD}^{4} \mid \mathrm{M}$. Angelyn Bethel $\mathrm{MD}^{5}$ | \\ John B. Buse MD, $\mathrm{PhD}^{6}$ | Jennifer B. Green $\mathrm{MD}^{7}$ | Keith D. Kaufman $\mathrm{MD}^{8}$ | \\ Helena W. Rodbard MD ${ }^{9}$ | Tsvetalina Tankova MD, DMedSci ${ }^{10}$ | \\ Cynthia M. Westerhout PhD ${ }^{3}$ | Eric D. Peterson MD, $\mathrm{MPH}^{7}$ | \\ Rury R. Holman MB ChB, FRCP, FMedSci ${ }^{5}$ | Paul W. Armstrong $\mathrm{MD}^{3}$; on behalf of the \\ TECOS Study Group
}

\footnotetext{
${ }^{1}$ St. Michael's Hospital, University of Toronto, Toronto, Ontario, Canada

${ }^{2}$ Department of Medicine, University of Alberta, Edmonton, Alberta, Canada

${ }^{3}$ Canadian VIGOUR Centre, University of Alberta, Edmonton, Alberta, Canada

${ }^{4}$ Wolfson Diabetes and Endocrine Clinic, Addenbrooke's Hospital, Cambridge University Hospitals Foundation Trust, Cambridge, UK

${ }^{5}$ Diabetes Trials Unit, Oxford Centre for Diabetes, Endocrinology and Metabolism, University of Oxford, Oxford, UK

${ }^{6}$ University of North Carolina School of Medicine, Chapel Hill

${ }^{7}$ Duke Clinical Research Institute, Duke University School of Medicine, Durham, North Carolina

${ }^{8}$ Merck \& Co., Inc., Kenilworth, New Jersey

${ }^{9}$ Endocrine and Metabolic Consultants, Rockville, Maryland

${ }^{10}$ Clinical Centre of Endocrinology, Medical University, Sofia, Bulgaria

Corresponding Author: Robert G. Josse MBBS, St. Michael's Hospital, University of Toronto, 61 Queen Street East, 6th Floor, Toronto, Ontario M5C 2 T2, Canada (josserg@smh.ca).

Funding Information

Funded by Merck Sharp \& Dohme, a subsidiary of Merck \& Co., Inc.
}

\begin{abstract}
Aim: To examine fracture incidence among participants in the Trial Evaluating Cardiovascular Outcomes with Sitagliptin (TECOS).

Research design and methods: We used data from 14671 participants in the TECOS study who were randomized double-blind to sitagliptin $(n=7332)$ or placebo $(n=7339)$. Cumulative fracture incidence rates were calculated and their association with study treatment assignment was examined using multivariable Cox proportional hazards regression.

Results: The baseline mean (standard deviation) participant age was 65.5 (8.0) years, diabetes duration was 11.6 (8.1) years and glycated haemoglobin level was 7.2 (0.5)\% [55.2 (5.5) mmol/ $\mathrm{mol}$, and $29.3 \%$ of participants were women and 32.1\% were non-white. During 43222 person-years' follow-up, 375 (2.6\%; 8.7 per 1000 person-years) had a fracture; 146 were major osteoporotic fractures (hip, $n=34$; upper extremity, $n=81$; and clinical spine, $n=31$ ). Adjusted analyses showed fracture risk increased independently with older age $(P<.001)$, female sex $(P<.001)$, white race $(P<.001)$, lower diastolic blood pressure $(P<.001)$ and diabetic neuropathy $(P=.003)$. Sitagliptin, compared with placebo, was not associated with a higher fracture risk [189 vs 186 incident fractures: unadjusted hazard ratio (HR) 1.01, 95\% confidence interval $(\mathrm{Cl}) 0.82$ to $1.23, P=.944$; adjusted $\mathrm{HR} 1.03, P=.745$ ], major osteoporotic fractures $(P=.673)$ or hip fractures $(P=.761)$. Insulin therapy was associated with a higher fracture risk (HR 1.40,95\% Cl 1.02-1.91; $P=.035)$, and metformin with a lower risk (HR 0.76, 95\% Cl 0.59-0.98; $P=.035)$.

Conclusion: Fractures were common among people with diabetes in the TECOS study, but were not related to sitagliptin therapy. Insulin and metformin treatment were associated with higher and lower fracture risks, respectively.
\end{abstract}

\section{KEYWORDS}

diabetes, DPP-4 inhibitors, fractures, sitagliptin

\section{1 | INTRODUCTION}

Type 2 diabetes is associated with an elevated risk of fragility fractures, particularly peripheral fractures. ${ }^{1-3}$ The pathophysiology is multi-factorial, relating largely to changes in bone quality, which involves the pathology underlying diabetes complications including microvascular disease and glycosylation of bone proteins, especially collagen. ${ }^{4}$ There are also indirect mechanisms by which diabetes and 
its treatments may increase risk of fracture, such as via episodes of severe hypoglycaemia and/or increased incidence of falls. ${ }^{5,6}$ As diabetes is associated with a greater fracture risk, it is important to know whether drugs used to treat diabetes may influence that risk. ${ }^{7,8}$ In the international Diabetes Outcome Progression Trial (ADOPT), participants randomized to receive the thiazolidinedione (TZD) rosiglitazone had a higher incidence of peripheral fractures than those receiving placebo. $^{9}$

Incretin hormones [e.g. glucagon-like peptide (GLP)-1 and GLP-2] have multiple actions on bone, and influence the proliferation of mesenchymal progenitor cells. GLP-1 has been reported to induce osteoblast differentiation, to inhibit osteoclast activity and to stimulate bone formation in rodents. ${ }^{4}$ GLP-2, secreted after food ingestion, inhibits bone resorption. ${ }^{10}$ Both GLP-1 and GLP-2 are formed by the cleavage of the proglucagon molecule, and both are degraded by the dipeptidyl peptidase-4 (DPP-4) enzyme. ${ }^{11}$ Because DPP-4 inhibitors prevent the degradation of incretin hormones, they could be associated with beneficial effects on the skeleton. A recent meta-analysis of phase II and III DPP-4 inhibitor trials (28 studies conducted in 20000 patients) showed a $40 \%$ reduction in the risk of fractures compared with placebo $(P=.045)$; however, that analysis was based on only 63 fracture events. ${ }^{12}$ A secondary analysis over 2 years' follow-up from the placebo-controlled Saxagliptin Assessment of Vascular Outcomes Recorded in Patients with Diabetes MellitusThrombolysis in Myocardial Infarction 53 (SAVOR-TIMI 53) trial of saxagliptin (which was not part of the aforementioned DPP-4 inhibitor meta-analysis) showed no association between saxagliptin and fracture. ${ }^{13}$ Moreover, a recent large, population-based cohort study of people with type 2 diabetes found that the new use of sitagliptin was not associated with increased risk of fracture compared with non-users, ${ }^{14}$ consistent with two other studies. ${ }^{15,16}$

In the present study, we performed a prespecified exploratory intention-to-treat analysis of fracture data from the Trial Evaluating Cardiovascular Outcomes with Sitagliptin (TECOS), which was a large, global, prospective controlled trial of 14735 participants randomized to receive the DPP-4 inhibitor sitagliptin or placebo.

\section{RESEARCH DESIGN AND METHODS}

\section{1 | Trial overview}

The design, rationale and main results of TECOS have been published in detail elsewhere. ${ }^{17,18}$ Briefly, 14735 participants from 38 countries were randomized between December 2008 and July 2012. Eligible participants were aged $\geq 50$ years with type 2 diabetes, cardiovascular disease, and glycated haemoglobin ( $\mathrm{HbA1c}$ ) values of $6.5 \%$ to $8.0 \%$ (48$64 \mathrm{mmol} / \mathrm{mol}$ ) on stable-dose mono- or combination therapy with metformin, pioglitazone or sulphonylurea, or insulin with or without metformin. Study participants were randomized (1:1) in a double-blind fashion to either sitagliptin or placebo at dosing appropriate for their estimated glomerular filtration rate (eGFR). Patients with an eGFR $<30 \mathrm{~mL} / \mathrm{min}$ per $1.73 \mathrm{~m}^{2}$ were not eligible for enrolment. Treatment for diabetes and its comorbidities was provided by usual care providers based on local guidelines. The addition of any antihyperglycaemic agent, other than a GLP-1 receptor agonist or open-label DPP-4 inhibitor, was permitted, although use of rosiglitazone was discouraged. Data regarding the use of concomitant medications, occurrence of severe hypoglycaemia (hypoglycaemia requiring the assistance of another individual), death, hospitalization, cardiovascular events and interventions, diabetes complications, fractures, serious adverse events and adverse events resulting in study drug discontinuation were recorded at participant visits. An independent clinical events classification committee whose members were unaware of treatment assignments adjudicated all events of death, myocardial infarction, stroke, hospitalization for unstable angina or heart failure, acute pancreatitis and cancer. Adjudicated event definitions have been published previously. ${ }^{18}$ TECOS was designed and run by the Duke Clinical Research Institute (DCRI) and the University of Oxford Diabetes Trials Unit, in an academically independent collaboration with the sponsor, Merck Sharp \& Dohme Corp. The database was held at and independently verified by the DCRI. The protocol was approved by the ethics committees associated with all participating trial sites, and all participants provided written informed consent for trial participation. The trial was registered at ClinicalTrials. gov under the number NCT00790205.

\section{2 | Main trial results}

There were 14671 participants included in the intention-to-treat population with a median (interquartile range; maximum) follow-up of 3.0 (2.3-3.8; 5.7) years. Overall, $95.1 \%$ of participants allocated to sitagliptin and $94.1 \%$ allocated to placebo completed the study, with premature study medication discontinuation occurring in $26.1 \%$ and $27.5 \%$ of participants, respectively. Vital status was determined at study end for $97.5 \%$ of participants. The TECOS study showed sitagliptin to be non-inferior to placebo with respect to the primary composite endpoint of cardiovascular death, non-fatal myocardial infarction, non-fatal stroke, or hospitalization for unstable angina [hazard ratio (HR) 0.98 , 95\% confidence interval $(\mathrm{Cl}) 0.88$ to $1.09 ; P<.001$ for non-inferiority]. Random allocation was successful, and there were no clinically important, statistically significant differences between patients allocated to sitagliptin compared with patients allocated to placebo.

\section{3 | Fracture-related outcomes}

The primary outcome for this analysis was "any post-randomization incident fracture." Fracture data were collected prospectively using two complementary methods of ascertainment: first, as serious adverse events (typically these were events requiring hospitalization, such as a hip fracture or proximal humeral fracture requiring surgical fixation) that were captured using standard serious adverse event recording methods, ${ }^{17,18}$ and second, via an electronic case report form fracture questionnaire (Appendix S1) completed at follow-up visits by study personnel, when the presence and location of fractures were collected from patients. Information was ascertained regarding confirmation by a clinician and/or whether radiograph verification of the fracture had been performed. We combined these events and hereafter refer to them as "any fracture." Secondary endpoints included major osteoporotic fractures (i.e. of the hip, clinical spine, proximal humerus or distal radius). 


\section{4 | Other measurements}

Detailed sociodemographic, clinical, laboratory and medicationrelated information was collected at the time of study enrolment. We did not have all of the variables necessary to calculate fracture risk according to the World Health Organization FRAX tool (without bone mineral density data) or other tools, such as parental history of hip fracture or pre-randomization history of fragility fracture, but we did have data on age, sex, race, body mass index, chronic kidney disease, smoking status, antidiabetic medications (sitagliptin, metformin, sulphonylureas, thiazolidenediones and insulin) and cardiovascular medications.

TABLE 1 Selected baseline demographic, clinical and laboratory characteristics and baseline diabetes and cardiovascular related treatments: overall and according to the presence or absence of incident fracture

\begin{tabular}{|c|c|c|c|c|}
\hline & $\begin{array}{l}\text { All } \\
(\mathrm{N}=14671)\end{array}$ & $\begin{array}{l}\text { No fracture } \\
(\mathrm{n}=14296)\end{array}$ & $\begin{array}{l}\text { Fracture } \\
(n=375)\end{array}$ & $P$ \\
\hline Age, years & $65.5(8.0)$ & $65.4(8.0)$ & $68.2(7.9)$ & $<.001$ \\
\hline Age $\geq 65$ years & 7735 (53.9) & 7485 (53.5) & $250(67.6)$ & $<.001$ \\
\hline Age $\geq 75$ years & $2004(14.0)$ & $1920(13.7)$ & $84(22.7)$ & $<.001$ \\
\hline Female sex & $4297(29.3)$ & $4130(28.9)$ & $167(44.5)$ & $<.001$ \\
\hline White race & 9957 (67.9) & $9662(67.6)$ & $295(78.7)$ & $<.001$ \\
\hline Duration of diabetes $\geq 10$ years & 7611 (51.9) & $7377(51.6)$ & $234(62.4)$ & $<.001$ \\
\hline Qualifying HbA1c, \% & $7.2(0.5)$ & $7.2(0.5)$ & $7.2(0.5)$ & .141 \\
\hline Qualifying $\mathrm{HbA} 1 \mathrm{c}, \mathrm{mmol} / \mathrm{mol}$ & $55.2(5.5)$ & $55.2(5.5)$ & $55.2(5.5)$ & .141 \\
\hline Body mass index, $\mathrm{kg} / \mathrm{m}^{2}$ & $30.2(5.6)$ & $30.2(5.6)$ & $30.5(5.8)$ & .374 \\
\hline Systolic blood pressure, $\mathrm{mm} \mathrm{Hg}$ & $135.0(17.0)$ & $135.1(17.0)$ & $133.4(16.5)$ & .070 \\
\hline eGFR, $\mathrm{mL} / \mathrm{min} / 1.73 \mathrm{~m}^{2} 1$ & $74.9(21.1)$ & $74.9(21.0)$ & 71.9 (22.9) & .006 \\
\hline eGFR $<50 \mathrm{~mL} / \mathrm{min} / 1.73 \mathrm{~m}^{21}$ & $1369(9.3)$ & $1317(9.2)$ & $52(14.0)$ & .002 \\
\hline LDL cholesterol, mmol/L & 2.35 (1.49) & $2.36(1.51)$ & $2.22(0.88)$ & .127 \\
\hline Previous cardiovascular disease & $10863(74.0)$ & $10593(74.1)$ & $270(72.0)$ & .360 \\
\hline Previous myocardial infarction & $6255(42.6)$ & $6103(42.7)$ & $152(40.5)$ & .404 \\
\hline Previous $\mathrm{PCl}$ & $5714(39.5)$ & $5562(39.4)$ & $152(41.3)$ & .472 \\
\hline Previous CABG & $3664(25.0)$ & $3571(25.0)$ & $93(24.8)$ & .937 \\
\hline Previous cerebrovascular disease & $3588(24.5)$ & $3478(24.3)$ & $110(29.3)$ & .026 \\
\hline Previous peripheral arterial disease & $2433(16.6)$ & $2367(16.6)$ & $66(17.6)$ & .592 \\
\hline More than one of the agents above & $8820(60.1)$ & $8604(60.2)$ & $216(57.6)$ & .313 \\
\hline$\beta$ blocker & $9322(63.5)$ & $9087(63.6)$ & $235(62.7)$ & .722 \\
\hline ACE inhibitor or ARB & $11555(78.8)$ & $11267(78.8)$ & $288(76.8)$ & .347 \\
\hline Calcium channel blocker & 4961 (33.8) & 4835 (33.8) & $126(33.6)$ & .929 \\
\hline Non thiazide diuretic & $2556(17.4)$ & $2482(17.4)$ & $74(19.7)$ & .232 \\
\hline Thiazide diuretic & $3464(57.5)$ & $3369(57.6)$ & $95(56.2)$ & .723 \\
\hline Aspirin & $11518(78.5)$ & $11223(78.5)$ & $295(78.7)$ & .940 \\
\hline Statin & $11719(79.9)$ & $11423(79.9)$ & $296(78.9)$ & .644 \\
\hline Ezetimibe & $761(5.2)$ & $742(5.2)$ & $19(5.1)$ & .915 \\
\hline Nitrates & $2813(19.2)$ & 2742 (19.2) & 71 (18.9) & .905 \\
\hline
\end{tabular}

Continuous variables are mean (standard deviation), and categorical variables are $n(\%)$.

Abbreviations: ACE, angiotensin-converting enzyme; ARB, angiotensin-receptor blocker; CABG, coronary artery bypass graft; PCl, percutaneous coronary intervention.

${ }^{1}$ Modification of Diet in Renal Disease formula used to calculate eGFR. Site-reported values are presented. 


\section{5 | Statistical analysis}

Details on the randomization performed in the present study have been published. ${ }^{18}$ We undertook several prespecified analytical steps (all analyses were performed on the intention-to-treat population). First, we reported the number and frequency of fractures over the duration of study follow-up and calculated the incidence rate in terms of fractures per 1000 person-years of follow-up. If a patient had more than one fracture, we only considered the first event. Second, we used Kaplan-Meier survival analysis to plot the cumulative estimated incidence of fracture according to treatment assignment, and then tested for differences between sitagliptin and placebo using the log-rank test. Third, exploratory analyses were used to describe the baseline characteristics for the overall study cohort and for those with versus those without incident fracture, and differences were tested using chi-squared tests or Student's t-tests, as appropriate.

A multivariable model was then developed to assess the independent association between assigned study treatment (sitagliptin vs placebo) and time to first fracture; the models were adjusted for other potentially important baseline factors using Cox proportional hazards regression. Sociodemographic, clinical or medication-related variables were included based on clinical (literature review, expert opinion) and statistical significance (univariate tests with $P$ value $<.10$; Table 1); no automated covariate selection procedures were used. Linearity of the effect of continuous covariates in these models was verified using restricted cubic spline functions with five knots. Results of the final model are shown in Table 2, and an identical analytical approach was applied to generate the results shown in Figure 1. All first-order interactions with the study treatment assignment were tested; none achieved nominal statistical significance $(P<.10)$, and none were included in the final multivariable model. We used visual inspection of log-minus-log plots to determine whether there were any violations of the proportional hazards assumptions; none were detected. Estimates of associations with time to fracture are reported as HRs and $95 \% \mathrm{Cls}$.
We then undertook three sensitivity analyses. First, we examined the association of the study treatment assignment with (1) major osteoporotic fractures and (2) hip fractures as the dependent variable of interest rather than any incident fracture. Second, because of the well-known strong association of older age with fracture, we repeated analyses restricted to those aged $\geq 75$ years, as well as those aged $\geq 65$ years. Third, we included one additional covariate defined as "any post-randomization severe hypoglycaemic event" in the multivariable adjustment model to assess whether hypoglycaemia may have contributed to the fracture risk.

Unless otherwise defined, we considered a two-sided $P$ value of $<.05$ to be statistically significant, and did not correct for multiple testing. SAS version 9.4 (Cary, North Carolina) was used for all statistical analyses.

\section{3 | RESULTS}

The mean (standard deviation) participant age was 65.5 (8.0) years, diabetes duration was 11.6 (8.1) years, and $\mathrm{HbA} 1 \mathrm{c}$ was $7.2(0.5) \%$ [55.2 (5.5) $\mathrm{mmol} / \mathrm{mol}$; $29.3 \%$ were women, $32.1 \%$ were non-white, $11.4 \%$ still smoked, and $22.6 \%$ reported diabetic neuropathy as a complication. Over a median (interquartile range) 3.0 (2.3-3.8) years of follow-up, $3.9 \%$ of participants had a non-fatal myocardial infarction, $2.1 \%$ had a non-fatal stroke, 3.1\% were hospitalized for heart failure, and $7.5 \%$ died. The baseline characteristics according to the presence or absence of incident fractures during the study are shown in Table 1.

\section{1 | Incidence of fractures}

During 43222 person-years of follow-up, 375 participants (2.6\%) had a fracture, giving an overall incidence rate of 8.7 per 1000 person-years. Of these fractures, 146 were major osteoporotic fractures (3.4 per 1000 person-years) and 34 were hip fractures (0.8 per 1000 person-years). Lower extremity fractures were the most commonly reported fractures ( $\mathrm{n}=102,2.4$ per 1000 person-years;

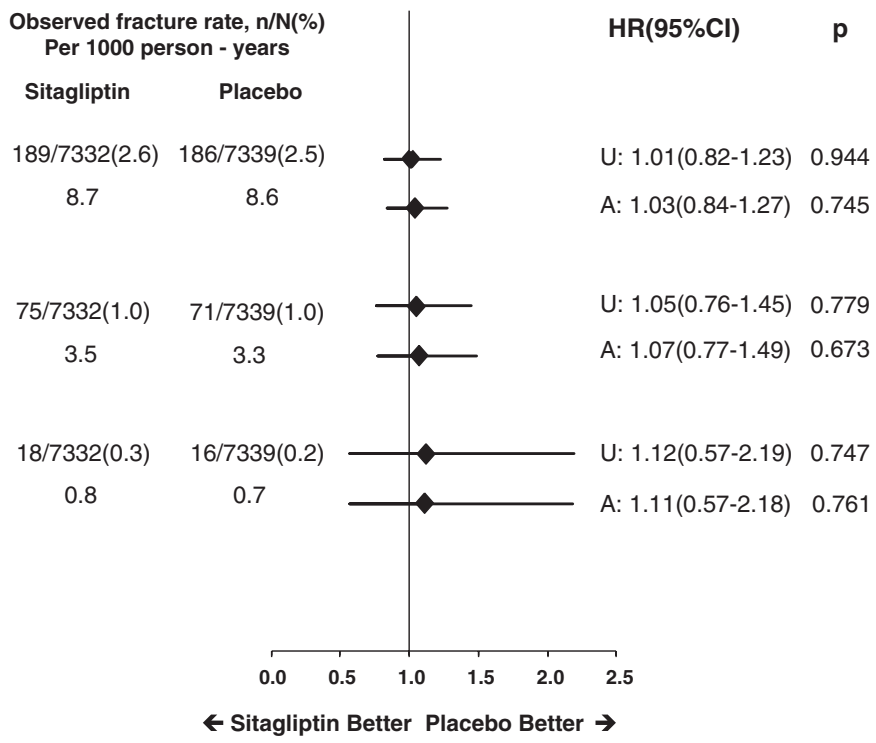

U: Unadjusted

A: Adjusted for age, sex, white race, diastolic blood pressure, current smoker, diabetes duration, diabetic neuropathy, and use of metformin, sulfonylurea, TZD, and insulin. 


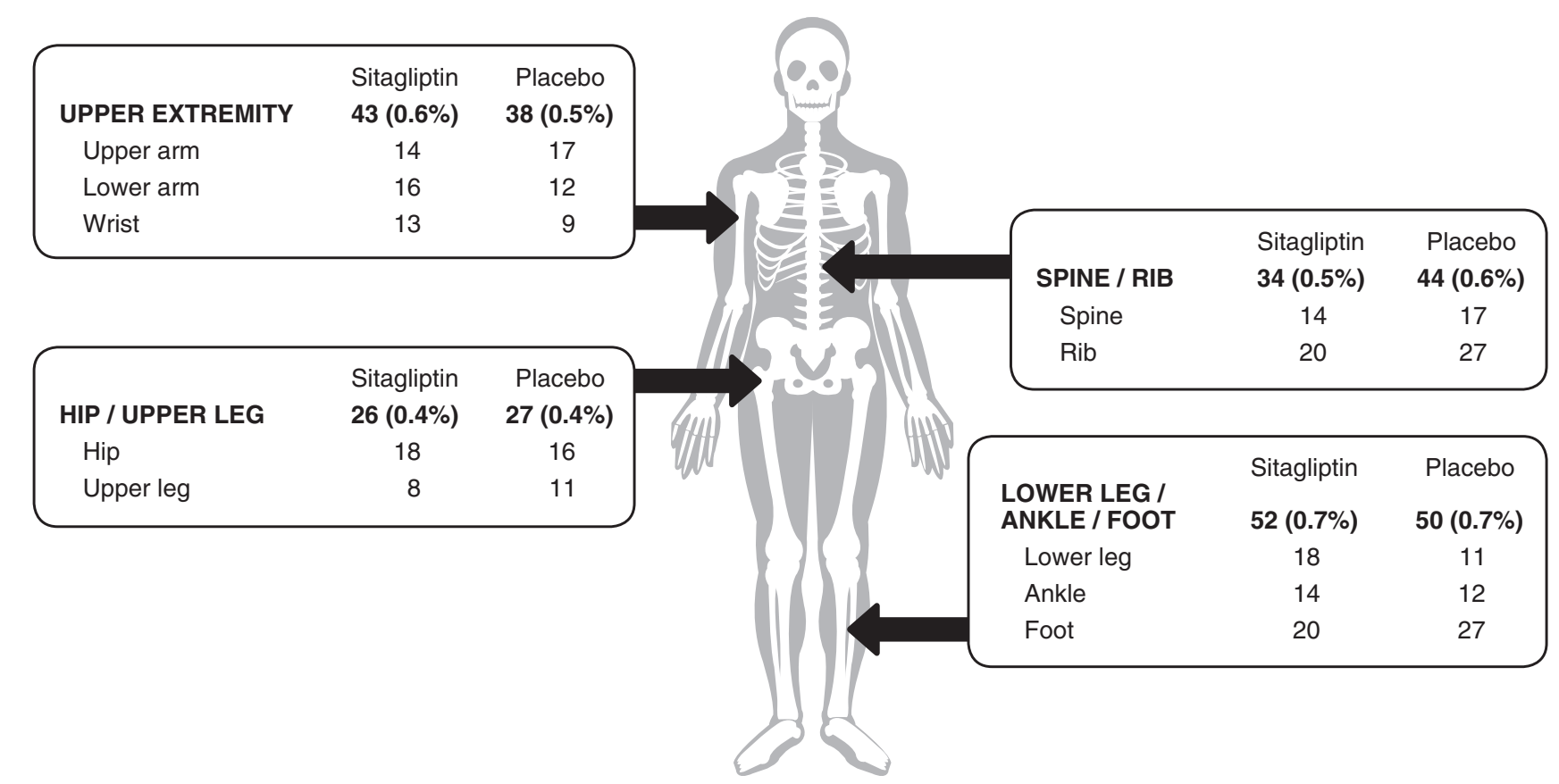

FIGURE 2 Frequency and location of incident fractures according to treatment assignment (sitagliptin vs placebo). Face, hand and other fractures excluded.

Figure 2). Those with an incident fracture were older and more likely to be female, to be white, to have a longer diabetes duration, and to have complications of diabetes and other comorbidities (Table 1). Of the 375 incident fractures, 132 (35\%) were ascertained (and thereafter confirmed by providers and radiographs) via the trial's routine adverse event reporting system, and an additional 243 (65\%) fractures were ascertained using the supplemental fracture survey of patients. Of the 243 fractures documented by survey, $91 \%$ were confirmed by providers, $83 \%$ were confirmed by radiographs, and $82 \%$ had confirmation by both providers and radiographs. Although the assigned study treatment was not associated with the hazard of fracture, older age, female sex, white race, lower diastolic blood pressure and diabetic neuropathy were each associated independently with an increased fracture risk in the adjustment model (Table 2).

\subsection{Association of diabetes treatments and fractures}

The majority of participants were taking metformin (81.6\%) at baseline, and $60.1 \%$ were taking two or more antidiabetic treatments (Table 1). After adjustment for sociodemographic and clinical factors, insulin therapy was associated with increased risk of fracture [adjusted HR 1.40 (95\% Cl 1.02-1.91); $P=.035$ ]; conversely, metformin use was significantly associated with a decreased risk of fracture [adjusted HR 0.76 (95\% Cl 0.59-0.98); $P=.035]$. TZD therapy was not associated with a significantly increased risk in adjusted analyses, but the $\mathrm{Cls}$ for this were wide, probably related to the low overall TZD usage in the trial [adjusted HR 1.54 (95\% Cl 0.94-2.52); $P=.087$ ].

\section{3 | Association of sitagliptin and fractures}

There were 189 incident fractures (8.7 per 1000 person-years) with sitagliptin compared with 186 fractures (8.6 per 1000 person-years) with placebo [unadjusted HR 1.01 (95\% Cl 0.82-1.23); $P=.944$ ] and curves of estimated cumulative fracture incidence essentially overlapped for the duration of the trial (Figure 3). Also, there was no significant association (in adjusted analyses) between assigned sitagliptin use and any incident fracture $(P=.745)$ or major osteoporotic $(P=.673)$ or hip fracture $(P=.761$; Figure 1$)$.

\subsection{Additional exploratory sensitivity analyses}

There were more fractures in the elderly participants, in both absolute and relative terms, in the present study. Just over half $(53.9 \%)$ of

TABLE 2 Association between assigned study treatment and time to first fracture in multivariable Cox regression model adjusted for other baseline factors

\begin{tabular}{ccc} 
& $\begin{array}{c}\text { Adjusted HR } \\
(95 \% \mathrm{Cl})\end{array}$ & $\mathbf{P}$ \\
\hline Assigned study treatment & & \\
Sitagliptin (vs placebo) & $1.03(0.84-1.27)$ & .749
\end{tabular}

\begin{tabular}{|c|c|c|}
\hline \multicolumn{3}{|l|}{ Adjustment variables } \\
\hline $\begin{array}{l}\text { Metformin therapy at } \\
\text { randomization }\end{array}$ & $0.76(0.59-0.98)$ & .035 \\
\hline $\begin{array}{l}\text { Sulphonylurea therapy at } \\
\text { randomization }\end{array}$ & $0.91(0.70-1.18)$ & .470 \\
\hline TZD therapy at randomization & $1.54(0.94-2.52)$ & .087 \\
\hline Insulin therapy at randomization & $1.40(1.02-1.91)$ & .035 \\
\hline Age (per year increase) & $1.03(1.02-1.05)$ & $<.001$ \\
\hline Female & $1.95(1.58-2.40)$ & $<.001$ \\
\hline White race & $1.58(1.23-2.04)$ & $<.001$ \\
\hline Current smoker & $1.16(0.83-1.62)$ & .399 \\
\hline Duration of diabetes $\geq 10$ years & $1.25(0.98-1.57)$ & .068 \\
\hline $\begin{array}{l}\text { Diastolic blood pressure }<80 \mathrm{~mm} \\
\mathrm{Hg}\end{array}$ & $1.50(1.21-1.86)$ & $<.001$ \\
\hline Diabetic neuropathy & $1.42(1.12-1.76)$ & .003 \\
\hline
\end{tabular}


the participants were aged $\geq 65$ years, and $14 \%$ were aged $\geq 75$ years [HR adjusted for age $\geq 65$ vs $<65$ years 1.50 (95\% Cl 1.20-1.88), $P=.001 ;$ HR adjusted for age $\geq 75$ vs $<75$ years $1.55(95 \% \mathrm{Cl} 1.21$ 1.99), $P=.001$ ]. For both age thresholds, fractures were balanced between sitagliptin and placebo. There were 129/3859 fractures (3.3\%) in participants aged $\geq 65$ years taking sitagliptin (5.8 per 1000 person-years) and $121 / 3876$ (3.1\%) in participants aged $\geq 65$ years on placebo (5.4 per 1000 person-years) for an adjusted HR of 1.07 (95\% $\mathrm{Cl} 0.84-1.38 ; P=.574)$. There were $44 / 970$ fractures $(4.5 \%)$ in participants aged $\geq 75$ years taking sitagliptin (16.9 per 1000 person-years) and $40 / 1034(3.9 \%)$ in participants aged $\geq 75$ years on placebo (14.3 per 1000 person-years) for an adjusted HR of 1.25 (95\% Cl 0.81 $1.92 ; P=.319)$.

Finally, after randomization, severe hypoglycaemic events were not uncommon, although there was no difference between sitagliptin and placebo [2.2\% vs 1.9\%; HR 1.12 (95\% Cl 0.89-1.40); $P=.330$ ]. In a sensitivity analysis, any severe hypoglycaemic event was independently associated with an increased risk of fracture [adjusted HR 1.85 (95\% Cl 1.14-2.99); $P=.012$ ].

\section{4 | DISCUSSION}

Among the complications associated with diabetes, fractures have been neglected despite an increased risk of $20 \%$ to $30 \%$ compared with patients without diabetes. ${ }^{3,19}$ In TECOS, fractures occurred at a rate of nine per 1000 person-years. Hence, the skeletal safety profiles of diabetic medications deserve attention. In this large, prospective, randomized, placebo-controlled trial of sitagliptin in patients with type 2 diabetes, we showed the following: fractures were common in this population; sitagliptin was not associated with a greater fracture risk; and metformin therapy was associated with a lower fracture risk, whilst insulin therapy was associated with a higher fracture risk.

It is difficult to assess fracture risk in people with type 2 diabetes, both in clinical trials and in practice. Bone mineral density is often not significantly lower in people with type 2 diabetes, and measurement of bone density in such people may not provide as useful an estimate of fracture risk as in those without diabetes. ${ }^{20}$ Type 2 diabetes has not yet been incorporated into the FRAX fracture risk assessment tool to try and improve its fracture risk prediction capability. ${ }^{21}$ Bone strength encompasses both bone quantity and bone quality, and it is particularly the latter that is thought to be compromised in people with diabetes. Some newer techniques such as trabecular bone score, a textural measurement derived from bone mineral density, may be more helpful in assessing fracture risk. ${ }^{22,23}$

There have been many preclinical and clinical studies looking at the effects of different antidiabetic agents on skeletal health., 4,8,24 Metformin, the most commonly used antidiabetic agent, either has a neutral or minor beneficial effect on bone mineral density and fracture risk, as demonstrated in this study, which is consistent with the Rochester population-based study ${ }^{25}$; however, as with other drugs, data are somewhat conflicting and incomplete. Similarly, on the basis of preclinical studies with incretins and DPP-4 inhibitors, it might be surmised that the incretin hormones would have beneficial effects on bone. This speculation was assessed in a recent meta-analysis of 16 randomized controlled trials with incretin agents whose results were generally neutral but included some divergent results depending on the GLP-1 receptor analogue used. The studies were, however, not designed as primary fracture outcome studies, so these conflicting results must be interpreted with caution. ${ }^{26,27}$ Nevertheless, we believe that the totality of randomized and non-randomized evidence (including the present study) strongly suggests that the DPP-4 inhibitor class of agents has no clinically important effect on bone or the risk of fracture.

The increased risk of fracture associated with insulin is noteworthy and has been reported previously. ${ }^{4,7,13,25}$ The pathophysiology as to why exogenous insulin might increase fracture risk is not well understood. From animal studies and assessment in type 1 diabetes, insulin is thought to be osteoanabolic. ${ }^{28}$ The situation in type 2 diabetes is more controversial and hampered by the lack of randomized trials directly assessing the effect of insulin on bone health. The use
FIGURE 3 Kaplan-Meier estimated cumulative incidence of any fracture according to treatment assignment (sitagliptin vs placebo).

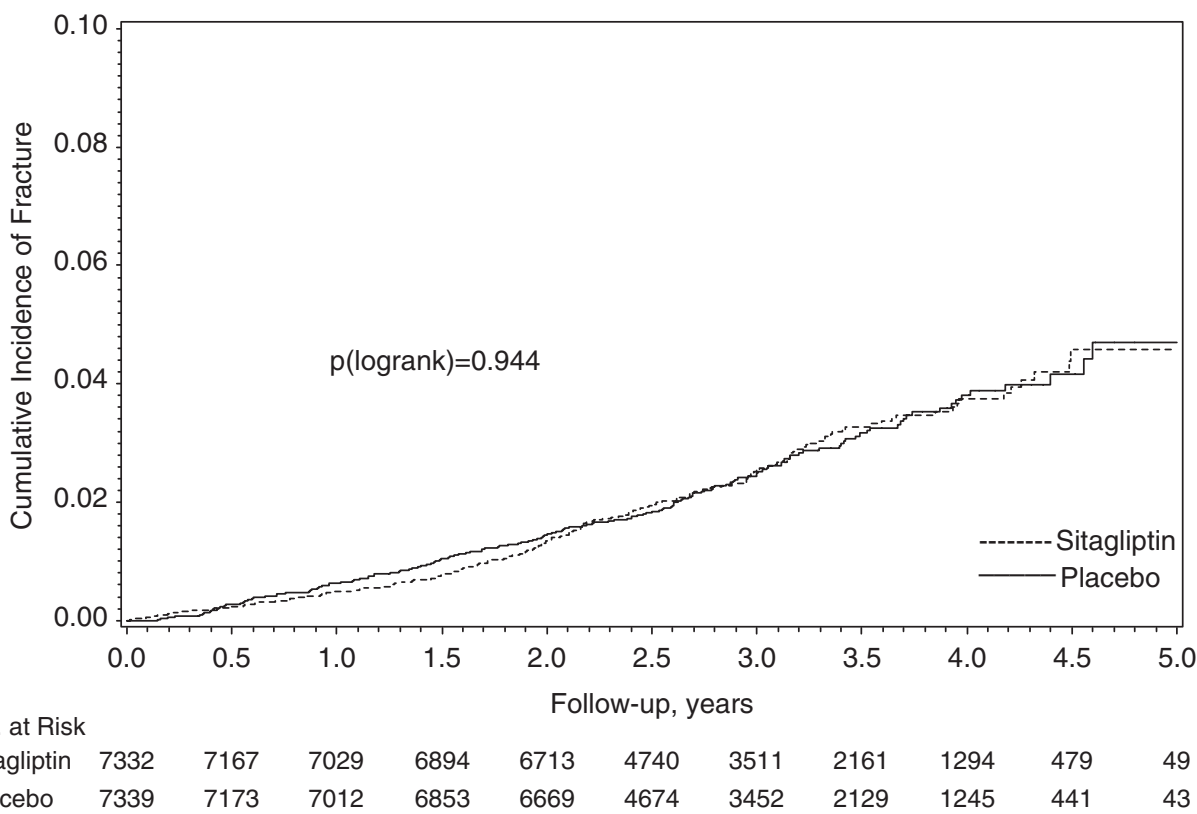


of insulin in type 2 diabetes may be a marker of more severe disease and the presence of more severe complications or an indication of a greater proclivity to symptomatic hypoglycaemia and falls. ${ }^{29}$ Previous studies have shown that falls are more common in patients with type 2 diabetes receiving insulin therapy and with lower $\mathrm{HbA} 1 \mathrm{c}$ levels. ${ }^{30-32}$ Even after correcting for falls, however, in these studies fracture risk remains increased. ${ }^{5,33-36}$ In a recent analysis from the Action to Control Cardiovascular Risk in Diabetes (ACCORD) trial, severe hypoglycaemia has been associated with falls and with fractures, in models adjusted for age, sex, race, treatment assignments and baseline $\mathrm{HbA1c}$ (unpublished data).

The present study supports both direct and indirect effects of insulin on fracture. In TECOS, where $2.2 \%$ of participants on sitagliptin and $1.9 \%$ on placebo experienced severe hypoglycaemia after randomization, there was also a significant association with fractures. Also, participants who experienced a fracture were more likely to have more advanced diabetes, as defined by the presence of complications (e.g. neuropathy), and to have more risk factors for falls (e.g. lower blood pressure). Even accounting for these factors in careful multivariable adjustment, insulin treatment remained associated with a higher risk of fracture, although the potential for residual confounding remains with any analysis of a non-randomized treatment. The pathophysiology relating diabetes, bone health and fracture is complex, and while an increased risk of injurious falls is probably an important (although not the only) contributor, the present findings suggest that some diabetes treatments have little or no effect on fracture, while other agents can directly or indirectly increase the risk. Clearly, this is an area that requires further investigation.

The present large prospective randomized controlled trial overcomes many of the problems inherent in observational studies, and provides a relatively homogenous population with detailed clinical information at baseline; however, it was powered for cardiovascular safety and not fracture risk, and any conclusions related to nonrandomized treatment assignments (e.g. analysis of risk associated with severe hypoglycaemic events) or post-randomization covariates are subject to the limitations of all observational studies. In addition, there were some design-specific limitations of our study.

First, with the methodology used, we were able to capture most symptomatic fractures, and self-report of recent fractures is valid and both very sensitive and specific ${ }^{33,34}$; however, certain fractures may have been missed, and we may have underestimated the overall fracture incidence. In general, only $\sim 25 \%$ of vertebral fractures are clinically apparent at the time of their occurrence-the majority are silent or occult ${ }^{37}$-but there is no reason to believe that the incidence of these fractures would differ according to antidiabetic treatments. Previous studies have shown that most fractures in people with type 2 diabetes are peripheral in location, and this is consistent with our findings (Figure 2).

Second, diabetes control in the population was reasonable, with mean baseline $\mathrm{HbA}_{1 \mathrm{c}}$ of 7.2 (0.5)\% [55.2 (5.5) $\left.\mathrm{mmol} / \mathrm{mol}\right]$, which was maintained fairly evenly throughout the study. Since glycaemic equipoise was achieved, we could not assess participants with very high or very low $\mathrm{HbA} 1 \mathrm{c}$ level, nor could we examine the role of excessively rapid changes in glycaemic control. We did examine postrandomization severe hypoglycaemic events as part of a prespecified analysis related to diabetes control. Beyond this one analysis, similar to that performed by others, ${ }^{13}$ we have refrained from an excessive number of speculative post hoc examinations of our data.

Third, we did not examine post-randomization risk factors for falls, such as new use of sedative-hypnotics, new initiations of antihypertensive agents, or new diagnoses of Parkinson's disease or stroke, and we did not have information related to the mechanisms of injury, such as whether fractures were low trauma. By the same token, we examined only baseline treatments and not post-randomization changes in doses, changes of regimen or medication adherence.

Last, the present study cannot provide insight into mechanism(s). We do not have detailed histories related to falls, bone mineral density tests, markers of bone turnover, or measures of "bone quality" based on biopsy or trabecular bone score. Our intent was to describe the rates and risk factors for fracture and the role of a randomized treatment versus placebo, and not to investigate the underlying pathophysiology.

In summary, the TECOS randomized study of the DPP-4 inhibitor sitagliptin, for which cardiovascular safety had already been demonstrated, found no increase in risk of fractures compared with placebo. We confirmed that several well-known clinical risk factors for fractures also increase risk in those with diabetes, and showed that risk factors for falls such as hypoglycaemia or hypotension predispose to fracture. However, we also found that insulin was associated with increased risk of fracture while metformin was associated with a decreased fracture risk. In contrast, sitagliptin was not associated with either an increased or decreased fracture risk. Because diabetes and osteoporosis and fractures are common chronic conditions, especially in the elderly, bone health should be considered an important aspect of diabetes care. The findings from the present study may increase awareness of this confluence of potentially preventable adverse events and help inform clinicians regarding the selection of diabetes treatments in people at high risk of fracture.

\section{ACKNOWLEDGMENTS}

The authors wish to thank the investigators, staff, and participants in the TECOS study, without whose efforts and collaboration this work would not have been possible. We thank the following academic partners and contract research organizations for their assistance: Parexel International; Jubilant Clinsys; Clinogent; Canadian VIGOUR Centre; Green Lane Coordinating Centre; and South Australian Health and Medical Research Institute. S.R.M. holds the Endowed Chair in Patient Health Management supported by the Faculties of Medicine and Dentistry and Pharmacy and Pharmaceutical Sciences at the University of Alberta. R.R.H. is an NIHR Senior Investigator. We acknowledge permission from the authors of reference 13 to use a similarly designed diagram to display fracture location.

\section{Conflict of interests}

R. G. J. has received grants or personal fees from Amgen, AstraZeneca, Boehringer-Ingelheim, Eli Lilly, Janssen and Merck. M. A. B. has received grants, personal fees, and other support from Merck, Sharp \& Dohme, other support from Boehringer-Ingelheim, Novo Nordisk, 
Glaxo and Smith Kline, and non-financial support from Bayer. J. B. B. has served as a consultant for PhaseBio, has received research support from AstraZeneca, Boehringer-Ingelheim, Bristol-Myers Squibb, Eli Lilly, GI Dynamics, J\&J, Lexicon, Novo Nordisk and Orexigen, is a shareholder in PhaseBio, and has been an advisor under contract with employer for AstraZeneca, Dance Biopharm, Eli Lilly, Elycylex, GI Dynamics, Lexicon, Merck, Metavention, Novo Nordisk, Orexigen and vTv Therapeutics. J. B. G. has received grants from Merck Sharp \& Dohme, AstraZeneca and GlaxoSmithKline, grants and personal fees from Merck Sharp \& Dohme, other support from Boehringer-Ingelheim, and personal fees from Bioscientifica and The Endocrine Society. K. D. K. is an employee of Merck \& Co., Inc., the manufacturer of sitagliptin, and owns stock and stock options in Merck \& Co., Inc. H. W. R. has received research support and consulting fees from Astra Zeneca, BI, Janssen, Lilly, Merck, NovoNoridsk, Sanofi and Regeneron. T. T. has received grants and personal fees from Merck, Sharp \& Dohme, Boehringer-Ingelheim, Novo Nordisk, Sanofi, Eli Lilly, Novartis, Servier and AstraZeneca. E. D. P. has received grants and personal fees from Janssen, grants from Eli Lilly, and personal fees from AstraZeneca, Bayer and Sanofi. R. R. H. has received grants and personal fees from Merck, grants from Bayer, AstraZeneca, and Bristol-Myers Squib, personal fees from Amgen, Bayer, Intarcia, Novartis, Novo Nordisk, and other support from GlaxoSmithKline, Janssen and Takeda. P. W. A. has received grants, personal fees and non-financial support from Merck and grants from AstraZeneca. S. R. M., Y. Z., A. A. and C. M. W. have no disclosures.

\section{Author contributions}

R. G. J., S. R. M. and Y. Z. designed the study, performed the statistical analysis and interpretation, and drafted, reviewed, and approved the final manuscript. A. A., M. A. B., J. B. B., J. B. G., K. D. K., H. W. R., T. T., C. M. W. and E. D. P. reviewed, revised and approved the final manuscript. P. W. A. and R. R. H. were contributors to the study design and critically reviewed, edited and approved the final manuscript. R. G. J., R. R. H. and P. W. A. are the guarantors of this work and, as such, had full access to all of the data in the study and take full responsibility for the work as a whole, including the study design and integrity of the data.

Parts of this work were presented at the American Diabetes Association meeting in 2016.

\section{REFERENCES}

1. Vestergaard $P$. Discrepancies in bone mineral density and fracture risk in patients with type 1 and type 2 diabetes-a meta-analysis. Osteoporos Int. 2007;18:427-444.

2. Janghorbani M, Van Dam RM, Willett WC, Hu FB. Systematic review of type 1 and type 2 diabetes mellitus and risk of fracture. Am J Epidemiol. 2007;166:495-505.

3. Liao CC, Lin CS, Shih CC, et al. Increased risk of fracture and postfracture adverse events in patients with diabetes: two nationwide population-based retrospective cohort studies. Diabetes Care. 2014;37:2246-2252.

4. Shanbhogue VV, Mitchell DM, Rosen CJ, Bouxsein ML. Type 2 diabetes and the skeleton: new insights into sweet bones. Lancet Diabetes Endocrinol. 2016:4:159-173.
5. Schwartz AV, Hillier TA, Sellmeyer DE, et al. Older women with diabetes have a higher risk of falls: a prospective study. Diabetes Care. 2002;25:1749-1754.

6. Schwartz AV, Margolis KL, Sellmeyer DE, et al. Intensive glycemic control is not associated with fractures or falls in the ACCORD randomized trial. Diabetes Care. 2012;35:1525-1531.

7. Gilbert MP, Pratley RE. The impact of diabetes and diabetes medications on bone health. Endocr Rev. 2015;36:194-213.

8. Palermo A, D'Onofrio L, Eastell R, Schwartz AV, Pozzilli P, Napoli N. Oral anti-diabetic drugs and fracture risk, cut to the bone: safe or dangerous? A narrative review. Osteoporos Int. 2015;26:2073-2089.

9. Kahn SE, Zinman B, Lachin JM, et al. Rosiglitazone-associated fractures in type 2 diabetes: an analysis from A Diabetes Outcome Progression Trial (ADOPT). Diabetes Care. 2008;31:845-851.

10. Henriksen DB, Alexandersen $\mathrm{P}$, Bjarnason $\mathrm{NH}$, et al. Role of gastrointestinal hormones in postprandial reduction of bone resorption. J Bone Miner Res. 2003;18:2180-2189.

11. Drucker DJ, Nauck MA. The incretin system: glucagon-like peptide-1 receptor agonists and dipeptidyl peptidase- 4 inhibitors in type 2 diabetes. Lancet. 2006;368:1696-1705.

12. Monami M, Dicembrini I, Antenore A, Mannucci E. Dipeptidyl peptidase-4 inhibitors and bone fractures: a meta-analysis of randomized clinical trials. Diabetes Care. 2011;34:2474-2476.

13. Mosenzon O, Wei C, Davidson J, et al. Incidence of fractures in patients with type 2 diabetes in the SAVOR-TIMI 53 trial. Diabetes Care. 2015;38:2142-2150.

14. Majumdar SR, Josse RG, Lin M, Eurich DT. Does sitagliptin affect the rate of osteoporotic fractures in type-2 diabetes? Population-based cohort study. J Clin Endocrinol Metab. 2016;101:1963-1969.

15. Driessen JH, van Onzenoort HA, Henry RM, et al. Use of dipeptidyl peptidase- 4 inhibitors for type 2 diabetes mellitus and risk of fracture. Bone. 2014;68:124-130.

16. Driessen JH, van Onzenoort HA, Straupe-Linde J, et al. Use of dipeptidyl peptidase 4 inhibitors and fracture risk compared to use of other antihyperglycemic drugs. Pharmacoepidemiol Drug Saf. 2015;24:10171025.

17. Green JB, Bethel MA, Paul SK, et al. Rationale, design, and organization of a randomized, controlled Trial Evaluating Cardiovascular Outcomes with Sitagliptin (TECOS) in patients with type 2 diabetes and established cardiovascular disease. Am Heart J. 2013;166:983989.e7.

18. Green JB, Bethel MA, Armstrong PW, et al. Effect of sitagliptin on cardiovascular outcomes in type 2 diabetes. N Engl J Med. 2015;373:232-242.

19. Leslie WD, Rubin MR, Schwartz AV, Kanis JA. Type 2 diabetes and bone. J Bone Miner Res. 2012;27:2231-2237.

20. Giangregorio LM, Leslie WD, Lix LM, et al. FRAX underestimates fracture risk in patients with diabetes. J Bone Miner Res. 2012;27:301-308.

21. Kanis JA, Oden A, Johansson H, Borgstrom F, Strom O, McCloskey E. FRAX and its applications to clinical practice. Bone. 2009;44:734-743.

22. Kim JH, Choi HJ, Ku EJ, et al. Trabecular bone score as an indicator for skeletal deterioration in diabetes. J Clin Endocrinol Metab. 2015;100:475-482.

23. Leslie WD, Aubry-Rozier B, Lamy O, Hans D, Manitoba Bone Density Program. TBS (trabecular bone score) and diabetes-related fracture risk. J Clin Endocrinol Metab. 2013;98:602-609.

24. Lecka-Czernik B. Safety of anti-diabetic therapies on bone. Clin Rev Bone Miner Metab. 2013;11:49-58.

25. Melton LJ 3rd, Leibson CL, Achenbach SJ, Therneau TM, Khosla S. Fracture risk in type 2 diabetes: update of a population-based study. J Bone Miner Res. 2008;23:1334-1342.

26. Su B, Sheng $H$, Zhang $M$, et al. Risk of bone fractures associated with glucagon-like peptide-1 receptor agonists' treatment: a meta-analysis of randomized controlled trials. Endocrine. 2015;48:107-115.

27. Mabilleau G, Mieczkowska A, Chappard D. Use of glucagon-like peptide-1 receptor agonists and bone fractures: a meta-analysis of randomized clinical trials. J Diabetes. 2014;6:260-266.

28. Thrailkill KM, Lumpkin CK Jr, Bunn RC, Kemp SF, Fowlkes JL. Is insulin an anabolic agent in bone? Dissecting the diabetic bone for clues. Am J Physiol Endocrinol Metab. 2005;289:E735-E745. 
29. Johnston SS, Conner C, Aagren M, Ruiz K, Bouchard J. Association between hypoglycaemic events and fall-related fractures in Medicare-covered patients with type 2 diabetes. Diabetes Obes Metab. 2012;14:634-643.

30. Vestergaard P, Rejnmark L, Mosekilde L. Relative fracture risk in patients with diabetes mellitus, and the impact of insulin and oral antidiabetic medication on relative fracture risk. Diabetologia. 2005;48:1292-1299.

31. Nicodemus KK, Folsom AR, lowa Women's Health Study. Type 1 and type 2 diabetes and incident hip fractures in postmenopausal women. Diabetes Care. 2001;24:1192-1197.

32. Monami M, Cresci B, Colombini A, et al. Bone fractures and hypoglycemic treatment in type 2 diabetic patients: a case-control study. Diabetes Care. 2008;31:199-203.

33. Colditz GA, Martin P, Stampfer MJ, et al. Validation of questionnaire information on risk factors and disease outcomes in a prospective cohort study of women. Am J Epidemiol. 1986;123:894-900.

34. Ismail AA, O'Neill TW, Cockerill W, et al. Validity of self-report of fractures: results from a prospective study in men and women across Europe. European Prospective Osteoporosis Study Group. Osteoporos Int. 2000;11:248-254.

35. de Liefde II, van der Klift $M$, de Laet $C E$, van Daele PL, Hofman A, Pols HA. Bone mineral density and fracture risk in type-2 diabetes mellitus: the Rotterdam Study. Osteoporos Int. 2005;16:1713-1720.
36. Strotmeyer ES, Cauley JA, Schwartz AV, et al. Nontraumatic fracture risk with diabetes mellitus and impaired fasting glucose in older white and black adults: the health, aging, and body composition study. Arch Intern Med. 2005;165:1612-1617.

37. Kendler DL, Bauer DC, Davison KS, et al. Vertebral fractures: clinical importance and management. Am J Med. 2016;129:221.e1-e10.

\section{SUPPORTING INFORMATION}

Additional Supporting Information may be found online in the supporting information tab for this article. 